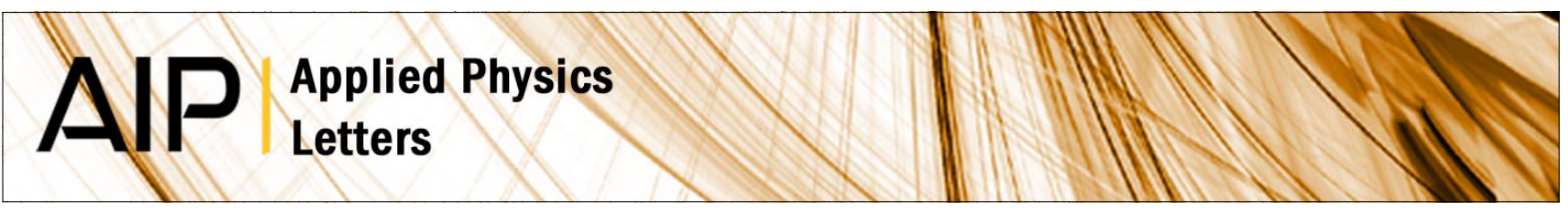

\title{
Surface modes for near field thermophotovoltaics
}

Arvind Narayanaswamy and Gang Chen

Citation: Appl. Phys. Lett. 82, 3544 (2003); doi: 10.1063/1.1575936

View online: http://dx.doi.org/10.1063/1.1575936

View Table of Contents: http://apl.aip.org/resource/1/APPLAB/v82/i20

Published by the American Institute of Physics.

Additional information on Appl. Phys. Lett.

Journal Homepage: http://apl.aip.org/

Journal Information: http://apl.aip.org/about/about_the_journal

Top downloads: http://apl.aip.org/features/most_downloaded

Information for Authors: http://apl.aip.org/authors

\section{ADVERTISEMENT}

\section{AIP Applied Physics Letters}

\section{EXPLORE WHAT'S NEW IN APL}

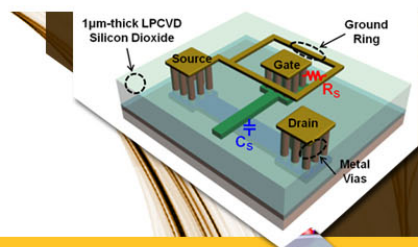

SURFACES AND INTERFACES

Focusing on physical, chemical, biological

structural, optical, magnetic and electrical

properties of surfaces and interfaces, and more...
ENERCY CONVERSION AND STORACE 


\title{
Surface modes for near field thermophotovoltaics
}

\author{
Arvind Narayanaswamy and Gang Chen ${ }^{\mathrm{a})}$ \\ Department of Mechanical Engineering, Massachusetts Institute of Technology, \\ Cambridge, Massachusetts 02139
}

(Received 21 January 2003; accepted 21 March 2003)

\begin{abstract}
Thermal radiative energy transfer between closely spaced surfaces has been analyzed in the past and shown not to obey the laws of classical radiation heat transfer owing to evanescent waves and, more recently, electromagnetic surface modes. We have analyzed the energy transfer between layered media, one of the layers being the thermal source, using a Green's functions method and the fluctuation-dissipation theorem. Based on the analysis, we propose a structure that can utilize the surface modes to increase the power density and efficiency of low temperature thermophotovoltaic generators. (C) 2003 American Institute of Physics. [DOI: 10.1063/1.1575936]
\end{abstract}

The effect of evanescent waves on the radiative energy transfer between two half planes has been studied in the past. ${ }^{1-3}$ It is well known from these studies that tunneling of evanescent waves can increase the radiative energy transfer above Planck's law. The potential for utilizing this increase for thermophotovoltaic (TPV) applications has also been studied. $^{3-5}$ The tunneling of evanescent waves, however, is a broadband phenomenon that favors long wavelength photons below the band gap of the photovoltaic (PV) material. More recently, the effect of electromagnetic surface excitations ${ }^{6}$ on the energy density in the near field of a half plane of silicon carbide $(\mathrm{SiC})$, a material which can support surface phonon polaritons, adjacent to vacuum has been studied. ${ }^{7-9}$ Thermal near fields in such cases have been shown to exhibit not only an increase of energy density but also greater spatial coherence and narrower bandwidth as compared to blackbody radiation. In this letter, we propose and analyze a structure to make use of the pseudomonochromatic nature of the energy transfer due to thermally excited surface waves and the near field effect to improve the performance of TPV generators. Because the efficiency of a TPV generator increases for narrow-band radiators, ${ }^{10,11}$ we expect that the transfer due to surface modes can be used to increase not only the power density but also the efficiency of the TPV generator.

We have analyzed the radiative energy transfer between layered media, with one of the layers being the thermal source, using the method of Green's functions. ${ }^{12}$ The source is assumed to be a half plane, adjacent to which are layers of different media, each characterized by a dielectric function (we consider only nonmagnetic materials). The Fourier component of the fluctuating electric field, $\boldsymbol{E}\left(\boldsymbol{r}_{1}, \omega\right)$, and magnetic field, $\boldsymbol{H}\left(\boldsymbol{r}_{1}, \omega\right)$, at any point, $\boldsymbol{r}_{1}$, outside a volume containing the sources is given by

$$
\begin{aligned}
& \boldsymbol{E}\left(\boldsymbol{r}_{1}, \omega\right)=i \omega \mu_{0} \int_{V} d^{3} r \overline{\overline{\boldsymbol{G}}}_{e}\left(\boldsymbol{r}_{1}, \boldsymbol{r}, \omega\right) \cdot \boldsymbol{J}(\boldsymbol{r}, \omega), \\
& \boldsymbol{H}\left(\boldsymbol{r}_{1}, \omega\right)=\int_{V} d^{3} r \overline{\overline{\boldsymbol{G}}}_{h}\left(\boldsymbol{r}_{1}, \boldsymbol{r}, \omega\right) \cdot \boldsymbol{J}(\boldsymbol{r}, \omega),
\end{aligned}
$$

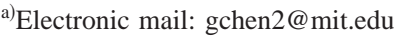

where $\overline{\overline{\boldsymbol{G}}}_{e}\left(\boldsymbol{r}_{1}, \boldsymbol{r}, \omega\right)$ and $\overline{\overline{\boldsymbol{G}}}_{h}\left(\boldsymbol{r}_{1}, \boldsymbol{r}, \omega\right)$, the dyadic Green's functions ${ }^{12}$ due to a point source at $\boldsymbol{r}$, are related by $\overline{\overline{\boldsymbol{G}}}_{h}\left(\boldsymbol{r}_{1}, \boldsymbol{r}, \omega\right)=\nabla_{1} \times \overline{\overline{\boldsymbol{G}}}_{e}\left(\boldsymbol{r}_{1}, \boldsymbol{r}, \omega\right) ; \boldsymbol{J}(\boldsymbol{r}, \omega)$ is the Fourier component of the current due to thermal fluctuations; and $\mu_{0}$ is the permeability of vacuum. The integration is performed over the entire volume $V$ containing the source. In order to compute the spectral Poynting vector at $\boldsymbol{r}_{1}$, we must compute the cross-spectral density of $E_{i}\left(\boldsymbol{r}_{1}, t\right)$ and $H_{j}\left(\boldsymbol{r}_{1}, t\right),\left\langle E_{i \omega} H_{j \omega}^{*}\right\rangle$, where the $*$ denotes the complex conjugate, the brackets denote a statistical ensemble average, and $i$ and $j$ refer to the three cartesian components $(i \neq j)$. In particular, the magnitude of the Poynting vector in the $z$ direction is given by $\frac{1}{2} \operatorname{Re}\left\langle E_{x \omega} H_{y \omega}^{*}-E_{y \omega} H_{x \omega}^{*}\right\rangle$. From Eq. (1), we can write an expression for $\left\langle E_{i \omega} H_{j \omega}^{*}\right\rangle$ as

$$
\begin{aligned}
\left\langle E_{i}\left(\boldsymbol{r}_{1}, \omega\right) H_{j}^{*}\left(\boldsymbol{r}_{1}, \omega\right)\right\rangle & \\
= & i \omega \mu_{0} \int_{V} d^{3} r \int_{V} d^{3} r^{\prime}\left\{G_{e_{i l}}\left(\boldsymbol{r}_{1}, \boldsymbol{r}, \omega\right)\right. \\
& \left.\times G_{h_{j m}}^{*}\left(\boldsymbol{r}_{1}, \boldsymbol{r}^{\prime}, \omega\right)\left\langle J_{l}(\boldsymbol{r}, \omega) J_{m}^{*}\left(\boldsymbol{r}^{\prime}, \omega\right)\right\rangle\right\} .
\end{aligned}
$$

With the expression for the Green's function and help of the fluctuation-dissipation theorem, ${ }^{13,14} \mathrm{Eq}$. (3) can be computed numerically. The fluctuation-dissipation theorem states that the cross-spectral density of different components of a fluctuating current source in equilibrium at a temperature $T$ is given by

$$
\left\langle J_{l}(\boldsymbol{r}, \omega) J_{m}^{*}\left(\boldsymbol{r}^{\prime}, \omega\right)\right\rangle=\frac{\epsilon_{0} \epsilon^{\prime \prime}(\omega) \omega \Theta(\omega, T)}{\pi} \delta_{l m} \delta\left(\boldsymbol{r}-\boldsymbol{r}^{\prime}\right),
$$

where $\epsilon^{\prime \prime}(\omega)$ is the imaginary part of the dielectric function of the source, $\epsilon_{0}$ is the permittivity of vacuum, and $\Theta(\omega, T)$ is given by $\hbar \omega /\left[\exp \left(\hbar \omega / k_{B} T\right)-1\right]$.

Polar materials such as $\mathrm{SiC}$, cubic boron nitride $(c \mathrm{BN})$, hexagonal boron nitride $(h \mathrm{BN})$, and boron carbide $(\mathrm{BC})$ have the ability to support surface phonon polaritons. In the frequency range of interest, the dielectric function can be expressed by the relation $\epsilon(\omega)=\epsilon_{\infty}\left(\omega^{2}-\omega_{\mathrm{LO}}^{2}+i \gamma \omega\right) /\left(\omega^{2}\right.$ $\left.-\omega_{\mathrm{TO}}^{2}+i \gamma \omega\right)$, where $\omega_{\mathrm{TO}}$ and $\omega_{\mathrm{LO}}$ are the transverse and logitudinal optical phonon frequencies, $\gamma$ is the damping fac- 
TABLE I. Values of $\omega_{\mathrm{LO}}, \omega_{\mathrm{TO}}, \gamma$ (in electron volts) and $\epsilon_{\infty}$ for $\mathrm{SiC}$ (see Ref. 16), BN (see Refs. 17 and 18), and BC (see Ref. 19).

\begin{tabular}{ccccc}
\hline \hline & $\omega_{\text {LO }}$ & $\omega_{\text {TO }}$ & $\gamma$ & $\epsilon_{\infty}$ \\
\hline $\mathrm{SiC}$ & 0.12 & 0.098 & $\approx 5.88 \times 10^{-4}$ & 6.7 \\
$c \mathrm{BN}$ & 0.1616 & 0.1309 & $\approx 6.55 \times 10^{-4}$ & 4.46 \\
$h \mathrm{BN}^{\mathrm{a}}$ & 0.1996 & 0.1695 & $\approx 6.59 \times 10^{-4}$ & 4.88 \\
$h \mathrm{BN}^{\mathrm{b}}$ & 0.1978 & 0.1872 & $\approx 9.92 \times 10^{-3}$ & 3.9 \\
$\mathrm{BC}$ & 0.1959 & 0.1352 & $\mathrm{c}$ & $\mathrm{c}$ \\
\hline \hline
\end{tabular}

${ }^{\mathrm{a}} \boldsymbol{E} \perp c$, electric field is perpendicular to optical axis.

${ }^{\mathrm{b}} \boldsymbol{E} \| c$, electric field is parallel to optical axis.

${ }^{\mathrm{c}}$ Not available.

tor, and $\epsilon_{\infty}$ is the high frequency dielectric constant. ${ }^{15}$ The values of $\omega_{\mathrm{LO}}$ and $\omega_{\mathrm{TO}}$ for $\mathrm{SiC},{ }^{16} \mathrm{BN},{ }^{17,18}$ and $\mathrm{BC}^{19}$ are listed in Table I.

For the case of energy transfer between two half planes of $c \mathrm{BN}$, one of them $(z<0)$ is at temperature $T(1000 \mathrm{~K})$ and the other $(z>\mathrm{d})$ is at room temperature $(300 \mathrm{~K})$, separated by a layer of vacuum of thickness $d$. The results of the analysis are plotted in Fig. 1. When the distance between the half planes is much larger than the characteristic wavelength $(3-10 \mu \mathrm{m})$, the spectral energy transfer between the two plates reaches a constant value. Notice that there is very little energy transfer between $\omega_{\mathrm{TO}}$ and $\omega_{\mathrm{LO}}$ at $d=1 \mathrm{~mm}$. As the distance between the two half planes is decreased, the shape of the spectral energy transfer curve begins to change drastically, the region between $\omega_{\mathrm{TO}}$ and $\omega_{\mathrm{LO}}$ becoming more and more prominent. At a spacing of $100 \mathrm{~nm}$, the peak spectral energy transfer, at $0.1565 \mathrm{eV}$, is close to three orders of magnitude larger than the energy transfer between two plane black surfaces at the same temperatures. This increase in energy transfer is because of the tunneling of fields due to the surface phonon polariton modes. The smaller peak that is observed at $0.1307 \mathrm{eV}$ is due to the tunneling of evanescent waves since the dielectric function near $\omega_{\text {TO }}$ takes on very large values.

This narrowband energy transfer phenomenon can be exploited for a TPV application by introducing a thin layer of absorbing PV material as shown in Fig. 2. The emitter, $c$ BN, is modeled as a half plane at a temperature $T(1000 \mathrm{~K})$. Layer A is a layer of vacuum, B is a layer of PV absorber at room temperature, and $\mathrm{C}$ is vacuum. The imaginary and real parts of the dielectric function of the PV material are assumed to be of the form

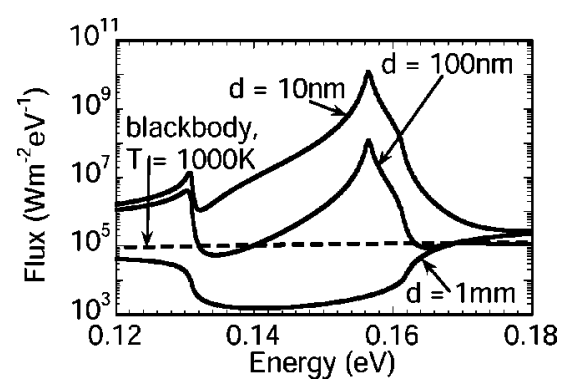

FIG. 1. Results of analysis of energy transfer between two half planes of $c \mathrm{BN}$.

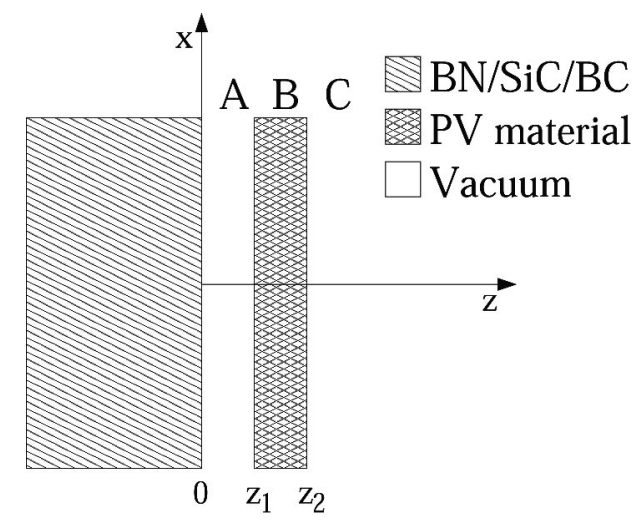

FIG. 2. Potential structure for TPV application.

$$
\begin{aligned}
& \epsilon_{i}(\omega)=\left\{\begin{array}{l}
A x^{-2} \sqrt{x-1}, x>1 \\
0, x<1
\end{array}\right. \\
& \epsilon_{r}(\omega)=\left\{\begin{array}{l}
B+A x^{-2}(2-\sqrt{1+x}), x>1 \\
B+A x^{-2}(2-\sqrt{1+x}-\sqrt{1-x}), x<1,
\end{array}\right.
\end{aligned}
$$

where $x=\hbar \omega / E_{g}$ and $A$ and $B$ are constants dependent on material properties. ${ }^{15}$ This form of the dielectric function is appropriate for direct band gap semiconductors. For the purpose of our calculation, we have chosen $\left(A, B, E_{g}\right)$ $=(6,10,0.13 \mathrm{eV})$.

The energy absorbed by the PV layer is calculated by computing the difference in the component of Poynting vector normal to the interfaces at $z=z_{1}$ and $z=z_{2}$. We have chosen the thickness of the PV layer, $z_{2}-z_{1}$, to be $100 \mathrm{~nm}$. For the assumed dielectric function, most of the flux above the band gap of the PV layer is absorbed. The results for varying thickness of the vacuum layer (layer A) are shown in Fig. 3. We see that as $z_{1}$ decreases, the flux absorbed by layer $\mathrm{B}$ increases in addition to becoming more narrowband in nature.

The total flux absorbed by the PV layer between 0.14 and $0.15 \mathrm{eV}$ is plotted in Fig. 4 as a function of the vacuum gap thickness. It is seen that the power absorbed at a vacuum gap of $20 \mathrm{~nm}$ is $117 \mathrm{~W} \mathrm{~cm}^{-2}$, almost three orders of magnitude higher than the solar insolation. At a vacuum gap of 100 $\mathrm{nm}$, the power absorbed is $4.86 \mathrm{~W} \mathrm{~cm}^{-2}$. While less than the power emitted by a blackbody source at $1000 \mathrm{~K}$ $\left(5.67 \mathrm{~W} \mathrm{~cm}^{-2}\right)$, the advantage is that the energy absorbed by the PV layer is in a much narrower bandwidth (full width half maximum is $\approx 2.5 \times 10^{-3} \mathrm{eV}$ as compared to $\approx 0.345 \mathrm{eV}$ for blackbody radiation at $1000 \mathrm{~K}$ ). While the



FIG. 3. Spectral flux absorbed by PV layer for $A=6, B=10, E_{g}$ $=0.13 \mathrm{eV}$, and $100 \mathrm{~nm}$ of PV layer. Solid (dashed) lines refer to flux (photon flux). $z_{1}$ is the vacuum gap. 


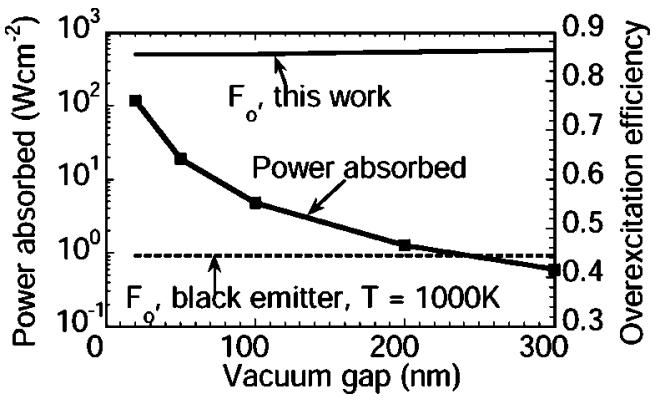

FIG. 4. Total flux absorbed by PV layer within the range $0.14-0.16 \mathrm{eV}$ and photon overexcitation efficiency $\left(F_{0}\right)$.

detailed efficiency analysis for the present TPV cell will be presented elsewhere, a plot of the photon overexcitation efficiency $\left(F_{0}\right)^{20}$ is shown in Fig. $4 . F_{0}$ is defined as the fraction of the energy absorbed by the PV layer that is usable. The value of $F_{0}$ is roughly 0.86 for this near monochromatic energy transfer while it is only 0.445 for a blackbody source at $1000 \mathrm{~K}$ and similar cell. This is an indication that in addition to improvements in power density, there could be improvements in efficiency as well. With $h \mathrm{BN}$ as the radiative layer, materials like InSb or $\mathrm{Hg}_{1-x} \mathrm{Cd}_{x} \mathrm{Te}$ can be potential materials for the PV layer. In the interest of brevity the results for $h \mathrm{BN}$ as emitter, which are similar to the results for $c \mathrm{BN}$, are not presented here.

In summary, we have implemented a general scheme based on a Green's function method and the fluctuationdissipation theorem to compute radiative energy transfer between layered media. We have used the earlier method to compute the spectral energy transfer between two half planes. In particular, we have seen that in materials that support electromagnetic surface waves, it is possible to see not only an enhancement but also a narrowband characteristic to the thermal energy transfer between the surfaces. While we have analyzed only materials supporting surface phonon polaritons, plasmon polaritons could also be used to produce a similar effect, generally at higher frequencues. To utilize surface plasmons, we should find the right materials which can support low loss surface plasmon polaritons in the frequency range around $0.3-0.8 \mathrm{eV}$ to match current TPV materials. We have analyzed the spectral energy transfer between a source material which can support surface waves and an absorbing PV layer. The energy transfer retains a part of the enhancement and narrowband characteristics that we have noticed between the two half planes. This effect can be used to improve the power density and efficiency of low temperature TPV generators.

The authors would like to thank J.-J. Greffet at Ecole Centrale, R. S. DiMatteo, M. Weinberg, and A. Meulenberg at Draper Lab, and P. Hagelstein at MIT for useful discussions. This work is supported by a DoD/ONR MURI on Electromagnetic Metamaterials through UCLA.

${ }^{1}$ D. Polder and M. V. Hove, Phys. Rev. 4, 3303 (1971).

${ }^{2}$ E. G. Cravalho, C. L. Tien, and R. P. Caren, J. Heat Transfer 89, 351 (1967).

${ }^{3}$ J. L. Pan, H. K. H. Choy, and C. G. Fonstad, IEEE Trans. Electron Devices 47, 241 (2000).

${ }^{4}$ R. S. DiMatteo, P. Greiff, S. L. Finberg, K. Young-Waithe, H. K. H. Choy, M. M. Masaki, and C. G. Fonstad, Appl. Phys. Lett. 79, 1894 (2001).

${ }^{5}$ M. D. Whale, Ph.D. thesis, MIT, 1997.

${ }^{6}$ D. L. Mills and E. Burnstein, Rep. Prog. Phys. 37, 817 (1974).

${ }^{7}$ R. Carminati and J.-J. Greffet, Phys. Rev. Lett. 82, 1660 (1999).

${ }^{8}$ A. V. Shchegrov, K. Joulain, R. Carminati, and J.-J. Greffet, Phys. Rev. Lett. 85, 1548 (2000).

${ }^{9}$ C. Henkel, K. Joulain, R. Carminati, and J.-J. Greffet, Opt. Commun. 186, 57 (2000).

${ }^{10}$ P. T. Landsberg and G. Tonge, J. Appl. Phys. 51, R1 (1980).

${ }^{11}$ M. A. Green, Prog. Photovoltaics 9, 257 (2001).

${ }^{12}$ L. Tsang, J. A. Kong, and K. H. Ding, Scattering of Electromagnetic Waves (Wiley, New York, 2000).

${ }^{13}$ L. D. Landau and E. M. Lifshitz, Statistical Physics (Addison-Wesley, Reading, MA, 1969).

${ }^{14}$ S. M. Rytov, Y. A. Kravtsov, and V. I. Tatarski, Principles of Statistical Radiophysics (Springer, Berlin, 1987), Vol. 3.

${ }^{15}$ P. Y. Yu and M. Cardona, Fundamentals of Semiconductors (Springer, Berlin, 1999).

${ }^{16}$ W. G. Spitzer, D. Kleinman, and J. Walsh, Phys. Rev. 113, 127 (1959).

${ }^{17}$ M. I. Eremets, M. Gauthier, A. Polian, J. C. Chervin, J. M. Besson, G. A. Dubitskii, and Y. Y. Semenova, Phys. Rev. B 52, 8854 (1995).

${ }^{18}$ R. Geick, C. H. Perry, and G. Rupprecht, Phys. Rev. 146, 543 (1966).

${ }^{19}$ Non-Tetrahedrally Bonded Binary Compounds II, edited by O. Madelung, Vol. III/17g of Landolt-Bornstein (1984), p. 36.

${ }^{20}$ P. F. Baldasaro, J. E. Raynolds, G. W. Charache, D. M. DePoy, C. T. Ballinger, and J. M. Borrego, J. Appl. Phys. 89, 3319 (2001). 